\title{
Intrapartum Shock
}

National Cancer Institute

\section{Source}

National Cancer Institute. Intrapartum Shock. NCI Thesaurus. Code C114373.

Severe loss of blood pressure leading to inadequate tissue perfusion after the onset of labor but prior to the delivery of the fetus. 